\title{
EL CONCEJO DE LACIANA EN EL SIGLO XVIII: ESTRUCTURA FAMILIAR Y ASISTENCIA SOCIAL
}

\author{
María José PÉREZ ÁLVAREZ \\ Universidad de León
}

\begin{abstract}
RESUMEN: La familia no es sólo un núcleo de reproducción, sino que es el centro en torno al cual gravitan toda una serie de comportamientos sociales tendentes al amparo y protección de todos sus miembros. El objetivo del trabajo será analizar el tipo de familia; el sistema de herencia, como factor vinculante a la expulsión de miembros útiles del hogar, o bien como medio de socorro a los más desamparados, ya sean parientes enfermos, célibes, ancianos, etc.
\end{abstract}

PALABRAS CLAVE: Concejo de Laciana, familia, asistencia social.

ABSTRACT: The family is not only an element of reproduction but also the core around which we can notice a sequence of social behaviours whose objective is to seek protection to all its members. The purpose of this work will be to analyse the type of family; the inheritance system as something linked with the expulsion of useful members out of home or as well as a way of giving aid to helpless people, including ill relatives, celibates, the elderly.

KEYWORDS: foreigners, blood cleansing, immigration, Vizcaya's ancient laws, trade, Vizcaya, Bilbao, xenophobia, $18^{\text {th }}$ and $19^{\text {th }}$ centuries.

\section{EL MODELO DE CORRESIDENCIA EN LA MONTAÑA DE LEÓN}

Del análisis de la unidad doméstica, atendiendo a las relaciones existentes entre los individuos que la componen, obtenemos las formas familiares externas ${ }^{1}$. Esta proyección social constituye un primer acercamiento al papel que la familia desempeña como agente de protección social de sus miembros ${ }^{2}$. En la montaña de

${ }^{1}$ El presente trabajo forma parte del proyecto Marginación y asistencia social en León durante la Edad Moderna, financiado por la Consejería de Educación de la Junta de Castilla y León (Ref.: LE 0270 A 07).

2 GOMILA GRAU, M.A. (1995): "Mecanismos de organización social a través de la familia. Tres municipios de Mallorca a finales del siglo XIX y XX". En GonZÁlez Portilla, M. y ZarragA 
León, en 1752, el tipo de familia más extendido era, evidentemente, el nuclear $(68,4 \%)$; a continuación aparecían las familias de tipo complejo (15\%), los solitarios $(13,2 \%)$-que engrosaban sus filas, fundamentalmente, con solteras, como consecuencia de las elevadas tasas de celibato femenino- y, finalmente, las carentes de estructura $(3,4 \%)^{3}$. Si ese análisis lo centramos en le Concejo de Laciana, la disponibilidad de cuatro padrones elaborados a lo largo del siglo XVIII 1718,1752,1761 y 1775-, nos han permitido conocer la dinámica de las fórmulas de corresidencia adoptadas en ese territorio en la centuria dieciochesca (cuadro $\mathrm{n}^{\circ} 1$ ).

En principio, la evolución parece ser la siguiente: partimos, en 1718, con los porcentajes más elevados del siglo de familia nuclear y compleja, y los más bajos en lo referente a solitarios y familias desestructuradas. Cuando llegamos a 1752, aumentan considerablemente las categorías simples, que, lógicamente, se detraen de las formas mayoritarias. Un panorama muy análogo lo encontramos en 1761. Pero en el último de los padrones, nos volvemos a colocar en valores muy similares a los de partida. Es decir, más del $25 \%$ de los hogares se reúnen en torno a las formas de familias de tipo extenso y múltiple, y, aunque no para volver a aquellos bajos niveles, experimentaron un retroceso las formaciones de solitarios y aquellas carentes de estructura. Si esta evolución la cruzamos con la trayectoria demográfica, y, por lo tanto, con las fases en las que se ejerció una mayor o menor presión sobre la tierra, el resultado sería el siguiente. Comenzamos el siglo XVIII con una montaña escasamente poblada, en la que se incardinan los estragos que causó la crisis de finales del siglo XVII con la sangría migratoria que resultó de ella. Por lo tanto el descenso de la presión demográfica daría lugar a alentadoras perspectivas en el campo de la disponibilidad de recursos. De hecho, la jerarquización socioeconómica que obtenemos antes de 1710 arroja los resultados más optimistas del siglo, con un tamaño medio de 4,1 Ha./por unidad campesina; e, incluso, el 50\% de las explotaciones estaban en condiciones de autoabastecerse. En ese contexto, la oportunidad de acceder a la tierra animaría la formación de nuevos hogares, por lo que el celibato y la viudedad perdieron espacio. A su vez, y teniendo en cuenta que las familias de tipo complejo van unidas a la disponibilidad económica ${ }^{4}$, es lógico el resultado que nos ofrece el padrón, un $28,5 \%$ de los

Sangroniz, K. (ed.). IV Congreso de la Asociación de Demografia Histórica. Vol. II, País Vasco: Universidad del País Vasco, pp. 653-673.

3 PÉrez Alvarez, M.J. (1996). La Montaña Noroccidental leonesa en la Edad Moderna. León: Universidad de Léon.

4 La pobreza debilita los vínculos familiares. CARASA SOTO, P. "La familia de los grupos populares próximos a la pobreza en la sociedad castellana decimonónica". Boletín de la Asociación de Demografía Histórica, 2/3, 1994, pp. 253-299, p. 258. 
hogares añadían a algún pariente. Es el momento secular en que las unidades de producción están más capacitadas para que dentro de ellas pueda garantizarse la reproducción social, y, por extensión, la asistencia.

Pero sobre los resultados de 1718 incidirían dos cuestiones que mediatizarían mucho los resultados que arroja el recuento en lo referente a la composición de las unidades domésticas. En primer lugar, la emigración que sufría el Concejo de Laciana. Es ésta una variable demográfica con gran repercusión sobre los hogares complejos. Debido a que la mayor parte de los emigrantes eran colaterales solteros, por lo que se verían muy disminuidas las probabilidades de formar unidades familiares de tipo extenso. Y, en segundo lugar, en 1718 la montaña acababa de salir de una importante crisis demográfica, con el consiguiente vacío demográfico y generacional que estas coyunturas acarrean. De ahí, que las posibilidades de aglutinar bajo un mismo techo tres generaciones, o formar hogares de tipo múltiple, en ese momento eran más limitadas que en otras coyunturas poblacionales.

La expansión demográfica que se vivió a lo largo del siglo XVIII, y que tuvo el momento de mayor euforia a mediados de la centuria, vino acompañado de un aumento de la presión sobre los recursos, de lo que dimanaría una movilidad social descendente que sufrirían sobre todo las capas intermedias. No era el momento más propicio para establecerse como nuevos agregados domésticos, ni tampoco para seguir añadiendo parientes al hogar. De lo que surgiría el constatado aumento de solitarios y el descenso de familias complejas. De esa readaptación de la composición familiar al tamaño de la explotación y sus necesidades, derivó el que un número relativamente importante de personas vieran como los mecanismos asistenciales naturales, que serían los proporcionados en el seno de la familia, se desvanecieran.

En 1775, de nuevo, se da una situación muy similar a la de principios de siglo, aunque no tuvo, ni mucho menos, la misma intensidad. Pero sí la suficiente como para dejarse sentir en las formaciones más simples, las cuales, después de ir aumentando representación en fechas precedentes, de nuevo se colocan en parámetros algo más bajos.

Cuadro $\mathrm{n}^{\circ} 1$. La tipología de la familia lacianiega

\begin{tabular}{lrrrrrrrr}
\hline & 1718 & $\%$ & 1752 & $\%$ & 1761 & $\%$ & 1775 & $\%$ \\
\hline Personas solas & 3 & 2,1 & 40 & 10,5 & 61 & 12,3 & 13 & 7,9 \\
Sin estructura & 3 & 2,1 & 17 & 4,5 & 33 & 6,7 & 6 & 3,7 \\
Nuclear & 97 & 67,4 & 244 & 64,0 & 281 & 56,9 & 102 & 62,2 \\
Extensa & 17 & 11,8 & 41 & 10,8 & 54 & 10,9 & 20 & 12,2 \\
Múltiple & 24 & 16,7 & 39 & 10,2 & 65 & 13,2 & 23 & 14,0 \\
\hline Total & 144 & 100 & 381 & 100 & 494 & 100 & 164 & 100 \\
\hline
\end{tabular}


Al margen de todas esas fluctuaciones que fue experimentando la tipología familiar, lo que es un hecho incuestionable es que la reproducción social dentro del propio hogar tuvo un papel destacado en Laciana ${ }^{5}, y$, en principio, parece que estuvo asociada a momentos de retroceso demográfico. Ahora bien, si realizamos los cálculos que nos propone Mikelarena Peña ${ }^{6}$ sobre el Censo de 1860 coincidente con una coyuntura demográfica expansiva-, para conocer la tipología familiar en ese momento, el balance que arrojan los datos lacianiegos, es el siguiente. El tamaño medio de la familia, en 1860, era de 4,97 miembros/hogar; el número de personas casadas y viudas por hogar, 1,73; el cociente de mujeres casadas y viudas por núcleo familiar, 1,02; y la cifra de adultos por hogar, 2,83. Según ésto, dos de los cuatro indicadores nos sitúan al Concejo de Laciana formando parte de la España troncal. Quedarían al margen, por no alcanzar la cifra de corte, los resultados que arrojan el segundo y tercer cálculo. En el segundo la media obtenida es inferior a dos, cifra que marca la frontera entre un tipo de familia $\mathrm{u}$ otro. Pero tengamos en cuenta que el mismo autor nos alerta sobre que la emigración masculina puede introducir un sesgo importante en esta cuantificación. Precisamente esa variable demográfica estuvo presente en el concejo a lo largo de

${ }^{5}$ Pero en ningún momento pueden compararse con los resultados, en este sentido, de la Galicia interior. Por el contrario son ligeramente más elevadas que en Liébana o del Oriente asturiano y muy similares a territorios concretos de la montaña del Principado. SAAVEdRa Fernández, P. (1988). "Casa y comunidad en la Galicia interior". Semata, vol. 2, pp. 85-143;. LóPEz IGLESIAS, F. (1999). El grupo doméstico en la Asturias del siglo XVIII. Oviedo: Real Instituto de Estudios Asturianos.LANZA GARCíA, R. (1988). Población y familia campesina en el Antiguo Régimen. Santander: Universidad de Santander. Teniendo en cuenta que en los concejos asturianos colindantes con el Concejo de Laciana, como fueron los de Cangas de Tineo o Degaña, la complejidad llegó a ser del 36\% y 40,7\%, respectivamente. A medida que descendemos hacia el sur de la provincia de León ese índice iba descendiendo. Laciana podría considerarse como una zona de transición entre dos modelos familiares. La familia troncal, característica del norte de la Península, y la familia nuclear, que modeló el centro y sur de España. Como ejemplos de la provincia de León, en el Bierzo, la complejidad estuvo entre el 3,7\% y 5,7\%; en la Vega del Esla, 3,4\%, en la Maragatería, prácticamente no existió. En Sahagún, fue del 5\%. BARTOlomé Bartolomé, J. (1996): Vino y viticultores en el Bierzo. Sociedad y estructuras económicas durante el siglo XVIII, León: Universidad de León, PÉrEZ GARCÍA, J. M., (1998): Un modelo social leonés en crecimiento. La vega del Esla en 1700 y 1850, León: Universidad de León; Rubio PÉrez, L. M., (1995): La Burguesía Maragata, León: Universidad de León; PÉrez Álvarez, M. J., (2004): "Familia y estrategias familiares en el marco de unas estructuras socioeconómicas tradicionales: el modelo de la Montaña Norocciental leonesa en la Edad Moderna". Revista de Demografia Histórica, XXII-I, pp. 121-147. Como modelo de familia nuclear pueden verse, entre los innumerables trabajos que se podrían citar, REHER, D., (1988). Familia, población y sociedad en la provincia de Cuenca, 1700-1970, Madrid: Siglo XXI; CHACÓN JIMÉNEZ, F., (1990). Historia social de la familia en España. Alicante: Instituto de Cultura Juan Gil-Albert, etc.

${ }^{6}$ Mikelarena PeÑa, F. (1992). "Las estructuras familiares en la España tradicional: geografía y análisis a partir del Censo de 1860". Boletín de la Asociación de Demografia Histórica, 3, pp. 1561. 
toda la Edad Moderna, y siguió estándolo en esas fechas. De hecho a partir de 16 años las cifras de hombres son mucho más bajas que las de mujeres. Por ser, casi exclusivamente, ese sexo el que tenía que buscar alternativas ocupacionales fuera del Concejo de Laciana., en los momentos en el que el tamaño de la familia sufría un ajuste derivado de las necesidades de la unidad de producción. Respecto al tercer cómputo, el resultado que arroja el Censo de 1860 está muy cerca de la frontera que delimita la familia troncal. Supera el 1, pero no llega a 1,075. Pero al ser relativamente elevado el resultado de dividir el número de personas mayores de 20 años entre el número de cédulas -cómputo que introduce una nueva probabilidad, y es la de no identificar familia compleja sólo con hogar múltiple sino también con familia extensa- la cifra obtenida en Laciana, nos advierte de que, dentro de ese tipo de hogares, el mayor peso recayó en las moradas de tipo extenso.

De todo lo expuesto hasta aquí, se deduce que la familia compleja estuvo bastante arraigada en el Concejo de Laciana, de hecho hay dos momentos en que supera claramente el $25 \%$, en 1718 y 1775 . Así mismo, y a pesar de que indudablemente sobre este tipo de formaciones domésticas repercutían las fluctuaciones demográficas y económicas, no son ellas las únicas que se resienten de esos procesos, sino que también salían muy afectados los grupos englobados en las categorías más simples. Esta evolución e involución cuantitativa en el comportamiento de las estructuras familiares será el mecanismo de respuesta que las comunidades consideran más oportuno para afrontar los diferentes ciclos poblacionales y económicos, a fin de afrontar con garantías la reproducción social ${ }^{7}$. Pero no hemos de olvidar que aunque hablemos de respuesta comunitaria, ésta no debe entenderse como un modo de proceder consensuado por el grupo de unidades domésticas que constituyen la comunidad, sino que el "agente activo" 8 de los cambios es cada una de las familias. Por lo tanto, esos comportamientos son el resultado de la confluencia de conductas reflexionadas individualmente por cada una de las cédulas ${ }^{9}$. Trayectoria que, por otro lado, veremos como en el Concejo de

${ }^{7}$ Ferrer I Alós, LL., (1995). "Notas Sobre el uso de la familia y la reproducción social”. Boletín de la Asociación de Demografía Histórica, I, pp. 11-29.

8 ReHer, D. y CAMPS, D. (1995). "Las economías familiares dentro de un contexto histórico comparado”. Revista Española de Investigaciones Sociológicas, $n^{\circ}$ 56, pp. 165-191

${ }^{9}$ Conocer los objetivos de esas cédulas, o de los individuos que la componen, y que a su vez se insertan en un colectivo, es lo que nos permitirá entender la elección de las estrategias y por qué se producen. CHACÓN JiMÉNEZ, F., (2001): "Historia de grupos: parentesco, familias, clientelas, linajes". En Castillo, S. y Fernández, R. (Coor.) Historia Social y Ciencias Sociales. Lleida: Mileno, pp. 165-183. 
Laciana se ajusta a la elasticidad descrita por E. Wolf ${ }^{10}$, cuando relaciona troncalidad y recursos económicos. Según el autor, las formaciones complejas no son las más idóneas en los territorios donde los recursos económicos son tan escasos que resultaría inoperativa la familia compuesta. Este es el caso de 1752, en que la presión sobre los recursos obligaba a la desmembración familiar. El exponente inverso sería 1718, cuando la relativa holgura económica permitió a los lacianiegos acoger bajo un mismo techo a más de una generación. El mismo autor continúa señalando que el orden troncal tampoco es el más adecuado en aquellos lugares donde la abundancia de recursos permite la formación de nuevas cédulas familiares, y precisamente, no fue ese el signo económico distintivo de la montaña en ningún momento del Antiguo Régimen. De ahí que en todos los padrones aparezca la familia nuclear acompañada de porcentajes relativamente importantes de hogares extensos y múltiples. Por lo tanto, fueron cuestiones coyunturales y no estructurales las que determinaron el avance o retroceso del hogar troncal lacianiego.

En otro momento, hemos podido estudiar la relación que existía, a mediados del siglo XVIII en el Concejo de Laciana, entre tipo de familia, edad del titular del hogar y nivel económico (cuadro $\mathrm{n}^{\circ} 2$ ). El padrón de 1718 nos permitirá ratificar parte de esas conclusiones, o bien, conocer cual fue el comportamiento 34 años antes, ante otras perspectivas demográficas. Decimos parte de las correlaciones porque los datos referentes al tamaño de las explotaciones que nos propone ese recuento son muy incompletos. En cuanto a las coincidencias, en ambos casos estamos ante hogares cuyos titulares no son precisamente personas jóvenes, ya que, tanto en 1718 como en 1752, en torno a las tres cuartas partes de las jefaturas de los hogares recaían en personas que superaban los 40 años ${ }^{11}$. A su vez, salvo en hogares formados por solitarios y los desestructurados, las edades medias que se obtienen en ambos recuentos, en función de la formula de corresidencia elegida, son muy similares. También en las dos muestras el mayor número de familias se aglutina en moradas encabezadas por personas que tiene entre 40 y 49 años.

${ }^{10}$ Wolf. E. (1982). Los Campesinos. Barcelona: Labor, pp. 83-107.

${ }^{11}$ Muestra del retraso en la edad al matrimonio en la Montaña, con respecto a la Vega del Esla, es que mientras allí el porcentaje de los hogares encabezados por personas menores de 30 años, era del $20 \%$; en la montaña, esa cifra es sólo del 3,5\%, en 1718, y del 7,2\%, en 1752, ascenso propiciado por el adelanto de la edad matrimonial. PÉREz GARCíA, J. M., (1998): Un modelo social leonés en crecimiento.... También, si comparamos los resultado de la montaña con los de la Galicia Occidental, en nuestro caso se observa un retraso a la hora acceder al a jefatura del hogar. FERNÁNDEZ CORTIZO, C., (1988): "En casa y compañía: grupo doméstico y estrategias familiares en la Galicia Occidental a mediados del siglo XVIII". Semata, vol. 2, pp. 145-165. Allí dos tercios de los cabeza de familia superaban los cuarenta años, mientras en esta zona leonesa ese resultado llega a ser del $75 \%$. 
Siguiendo con las similitudes, en el tramo de menores de treinta años tenían más peso las familias de tipo complejo, con un predominio de la extensa descendente, que las nucleares. Se trata de parejas que ya habían prestado asistencia a la generación precedente y ahora continuaban esa labor no desamparando a sus colaterales. De hecho, la media de 4,8 individuos/hogar, en 1718, teniendo en cuenta la edad al matrimonio en ese momento en la Montaña, no se podría alcanzar de ningún otro modo ${ }^{12}$. A partir de los treinta años ya comienzan a ganar espacio las formaciones nucleares, las cuales alcanzarán su techo, en 1718, en el tramo siguiente; y en 1752, cuando la jefatura del hogar recaiga en personas que se encontraban entre los 50 y 59 años ${ }^{13}$. Durante esos años, habían ido expulsando del hogar a todas esas personas que estaban bajo su responsabilidad, y los hogares quedarán reducidos a progenitores e hijos. De todas formas, se puede comprobar como esa tarea de protección sobre los hermanos/as, sobrinos/as u otros parientes, que se les encomendó a los cabeza de familia, aún hay casos que se prolongará, al menos, hasta 50-59 años. A partir de los 50-60 años, en ambos censos, se constata como iban a tomar posición los hogares de tipo complejo, lo cual repercutirá en el avance que refleja el tamaño medio de la familia con respecto a los tramos de edad anteriores. A esas edades, la asistencia a los descendientes y colaterales comienza a mezclarse con la que buscan los progenitores, ante su inminente ancianidad. Por lo que, poco a poco, los hogares múltiples descendentes iban desplazando a la categoría extensa descendente, Es el momento en que las necesidades asistenciales hacia la pareja anciana empiezan a ser patentes, y aprovechando que sus hijos comienzan a casarse ofrecen a alguno de ellos, normalmente al varón primogénito, la posibilidad de quedarse en casa para darles el relevo generacional.

Respecto a las diferencias, nosotros destacaríamos dos. La primera de ellas, es que en 1752, a pesar de ser el momento con menor índice de complejidad, el tamaño medio de la familia es ligeramente superior en el tramo de 40-49 años. Dicho "aparente desajuste" puede explicarse recurriendo a la demografía cualitativa. Esa media sería la consecuencia de que en la segunda mitad del siglo XVIII se percibe un adelanto de la edad al matrimonio en la mujer, que bien pudo iniciarse unos años antes. Esa precocidad matrimonial equivale a adelantar la entrada al mundo de la procreación. A su vez, las medias más elevadas, en lo referente al tamaño medio de la familia, que se constatan en el recuento de 1718 en los hogares encabezados por personas menores 40 años, se deberían a que, al estar

\footnotetext{
${ }^{12}$ En nuestro caso, el tamaño medio de la familia, en relación a la edad del cabeza de la misma, no sigue una progresión tan clara como en la Galicia interior. SAAVEdRA FERnándEZ, P., (1988): "Casa y comunidad en la Galicia interior", Semata, vol. 2, pp. 85-143..

${ }^{13}$ LanZa García, R. (1988). Población y familia campesina en el Antiguo Régimen........p. 141.
} 
moviéndonos con porcentajes más elevados de formaciones compuestas, dentro del hogar aún se iban arrastrando colaterales. La otra diferencia es la referente a hogares formados por solitarios, y es que si en 1752 aún hay varias moradas cuyo titular podría estar en fase de transito, en 1718, en cambio, parece que los pocos célibes que hay son definitivos.

Cuadro $\mathrm{N}^{\mathrm{o}}$ 2. Tipología familiar, en función de la edad del cabeza de familia, en el Concejo de Laciana.

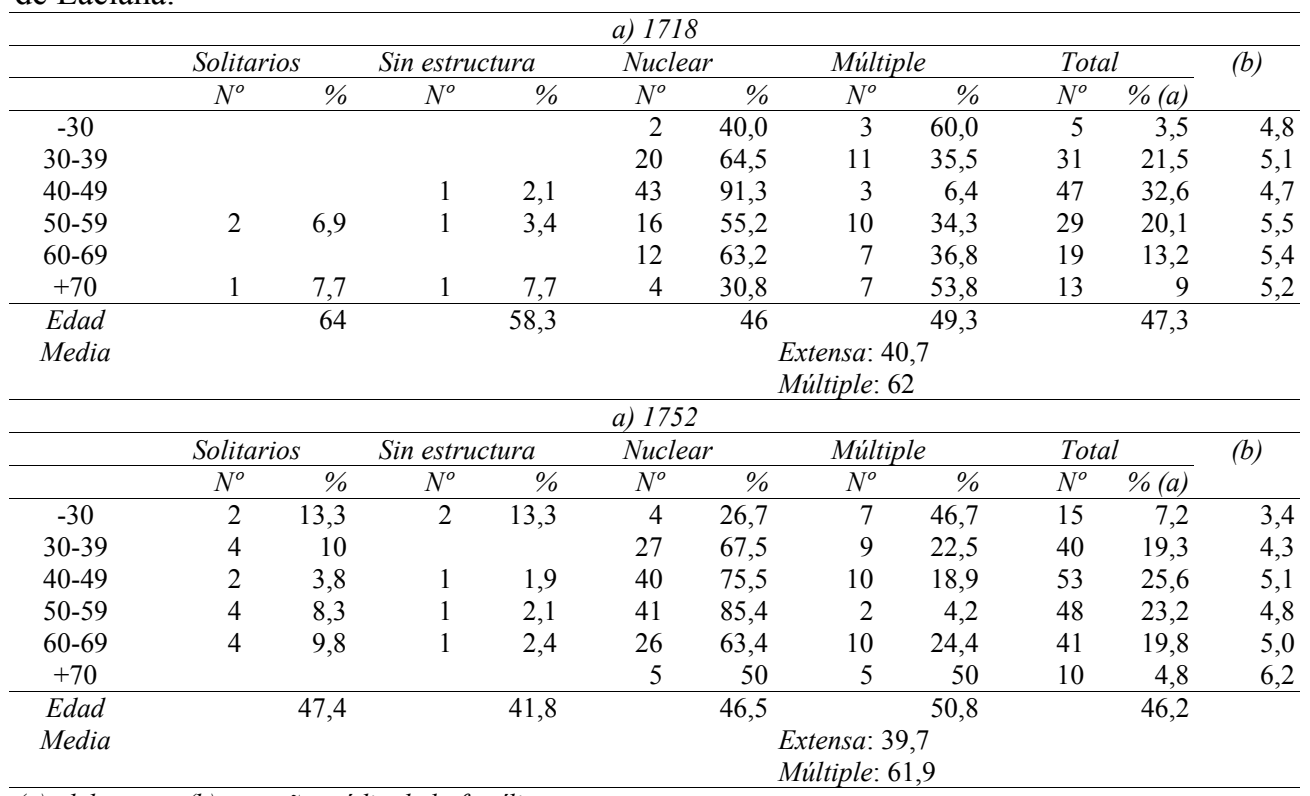

(a), del tramo; (b), tamaño médio de la família.

En el siguiente organigrama, hemos intentado representar los ciclos por los que atravesó entre el 21 y $28,5 \%$ de las familias del Concejo de Laciana ${ }^{14}$. El resto, al menos en teoría, tuvieron una evolución mucho más simple y, por consiguiente, pasaron por un número de fases más reducido. Para la elaboración del citado organigrama hemos tomado como referencia al hombre, ya que es a la casa del cónyuge masculino a donde se desplaza la mujer, desarrollándose en esa morada, a partir de entonces, el ciclo de un hogar múltiple. Por otro lado, y derivado de lo

${ }^{14}$ Insistimos en que se trata de un supuesto, aunque apoyado en datos reales, ya que: "la familia no es una organización monolítica ni está sujeta a una ley de evolución lineal o universal", como señaló FERnÁNDEZ CORTIZO, C., (1982): "A una misma mesa y manteles: la familia de Tierra de Montes”, Cuadernos de Estudios Gallegos, T. XXXIII, pp. 237-275. 
anterior, es también el varón, el que a cambio de la mejora, se hace cargo de sus hermanos. Las cifras medias utilizadas para la elaboración del gráfico han sido: edad al matrimonio del varón, 26,5 años; edad de defunción, 58,6; número medio de hijos, 4,1; intervalos intergenésicos, 19,3 meses desde el matrimonio hasta el nacimiento del primer hijo, 27,2 entre el alumbramiento del primogénito y el segundo hijo, 30,4 del segundo al tercero, y 29,7 entre el tercero y el cuarto ${ }^{15}$.

Organigrama $\mathrm{n}^{\mathrm{o}}$ 1. Supuesta evolución de un hogar complejo en la Montaña Noroccidental leonesa.

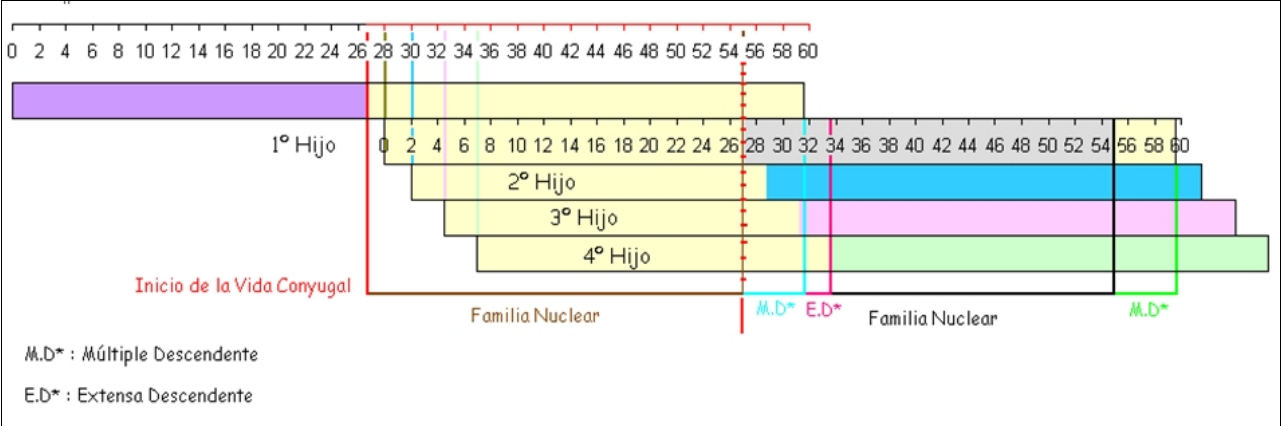

Antes de seguir adelante, es necesario señalar que el presente esquema es un modelo tipo, ya que no hemos de olvidar que las variables demográficas nupcialidad, natalidad, mortalidad y emigración- estaban sometidas a una gran inestabilidad, y, por lo tanto, el modelo podía mutar en pocos meses. El resultado de este ensayo fue el siguiente: partiendo de una familia nuclear un individuo cabeza de familia a lo largo de su vida detenta la jefatura en dos tipos de hogar: el nuclear y el múltiple descendente. Por el contrario, cuatro serán, al menos, los ciclos que recorre una persona que inició su vida conyugal en la casa paterna ${ }^{16}$ : múltiple descendente, extensa descendente, nuclear y múltiple descendente. En ambos podríamos añadir una tipología más si el cabeza de familia cede la administración en el hijo mejorado.

Respecto a la duración de cada una de esas etapas, comenzando por el patriarca, tenemos que si se casó a los 26,5 años, con algo más de 28 tendría su primer hijo,

15 Pérez Alvarez, M.J. (1996). La Montaña Noroccidental leonesa.... p.292

${ }^{16}$ En la comarca santanderina de Liébana, la mayor parte de los habitantes, a lo largo de su vida, en algún momento formaron parte de familias complejas., LANZA GARCíA, R. (1988). Población y familia campesina en el Antiguo Régimen....... 
con lo que inicia la subfase pareja con hijos, que será la más larga, dado que la etapa de crianza es la que más tiempo de vida absorbe a la pareja, y en la que permanecerá hasta que el primero de sus hijos contraiga matrimonio. Si éste se casa a la misma edad que su padre, la categoría de familia nuclear tuvo una duración de algo más de 28 años. Una vez que se produce el enlace del primogénito, el hogar patriarcal pasará a ser múltiple descendente, situación que se mantendría unos cinco años, hasta el fallecimiento del progenitor, y en ese tiempo, éste, aún podrá casar a otros dos se sus vástagos. Esta época puede acortarse o alargarse. Será más breve si el primogénito no es un varón, ya que, precisamente, no es la mujer el sexo escogido para continuar la línea familiar en la morada paterna; y se alargará si el hijo se casa a una edad más temprana. En cualquier caso, la variación media puede ser de unos dos-tres años arriba o abajo, ya que la precocidad matrimonial no fue el signo distintivo de la montaña; pero la variación real será mucho más amplia, ya que vendría determinada por la longevidad de todos los implicados en el proceso.

Una vez fallecido el patriarca, el primogénito, que relevará a su antecesor en la jefatura del hogar, tendrá que hacerse cargo de los hermanos/as que aún permanecen en casa, por lo que inauguramos una etapa de familia extensa descendente. Duraría unos dos o tres años, en condiciones medias, o mejor óptimas, es decir, suponiendo que todos sus hermanos, de una forma u otra casándose, emigrando..- salieran del hogar paterno. En esta fase familiar las variaciones pueden ser mucho mayores, y en lugar de durar dos-tres años, el ciclo podría alargarse considerablemente si lo que tiene son hermanas, más expuestas al celibato que los hombres ${ }^{17}$. En cualquier manera, la fase nuclear del primogénito, sería mucho más corta que la disfrutada por su padre, y condiciones medias, se reduciría a unos 22 años, porque en torno a los 55 casará a su primer hijo, y la familia entonces se convertirá en múltiple descendente.

\section{LAS ESTRATEGIAS ASISTENCIALES.}

La familia no es sólo un núcleo de reproducción, sino que es el centro en torno al cual gravitan toda una serie de comportamientos sociales tendentes al amparo y

${ }^{17}$ Por otro lado, no hemos de olvidar que en el Concejo de Laciana, la media de hijos que cada matrimonio llegaba a casar era de 1,9, con valores que oscilaban entre el 1,6, de las pequeñas explotaciones, y 2,7, entre los que detentaban los mayores patrimonios. PÉrez Álvarez, M. J., (2004). "Familia y estrategias familiares en el marco de unas estructuras socioeconómicas tradicionales: el modelo de la Montaña Norocciental leonesa en la Edad Moderna", Revista de Demografia Histórica, XXII-I, pp. 121-147. Por lo tanto, no es extraño que nos encontremos con elevados porcentajes de familia extensa descendente, lo que, a suvez, será la causa de que esa fase familiar se prolongue en el tiempo. 
protección de todos sus miembros. Esa asistencia tiene varias fases, que se manifiestan en una conducta de tipo circular en la que, en el caso de la familia troncal, siempre se ven implicadas tres generaciones. La primera, que se correspondería con la tercera del círculo anterior, escoge la formación de una tipología determinada cuando algún miembro de la segunda generación inicia su vida postnupcial. Fórmula corresidencial que estará relacionada con la forma asistencial que los progenitores hubieran escogido para su ancianidad, es decir, acogen, o no, en su seno a ese hijo/a a cambio de una sustancial porción de la herencia. Trato que beneficiará a ambas partes. A su vez, la segunda generación, que convive con sus progenitores, y que será la que alumbre a la tercera, tendrá una doble misión asistencial: sus padres y sus hijos. Hijos que cerrarán ese primer círculo cuando sus padres determinen, como anteriormente lo habían hecho sus abuelos, en qué tipo de hogar quieren que acontezcan los últimos años de su vida.

Dos son los momentos del ciclo vital donde los cuidados son imprescindibles, la infancia y la senectud. En el primer caso, son los padres los que incuestionablemente se encargarán de cubrir las necesidades básicas del periodo de crianza del niño: alimentación, alojamiento... .Las personas que en este caso son objeto de asistencia serán individuos pasivos, es decir, todo lo que en ellos revierte se lo proporcionan sus parientes más directos sin que ellos medien en el proceso. Muy distinto será cuando hablamos de asistencia y senectud, entonces los que reciben, o no, los cuidados son sujetos activos, es decir, ellos intervienen directamente para delimitar el marco en el cual quieren que se lleve a cabo el auxilio. Ahora bien, cuando decíamos que intervienen para solicitar asistencia ¿no tendríamos que decir "comprar asistencia"?. Transacción en la que, lógicamente tendrían ventaja los más favorecidos económicamente. Es indudable que los lazos afectivos y la solidaridad parental intervendrían en el proceso, pero éstos al no estar mediatizados por una cuestión económica el rastro documental que nos dejaron fue mucho mas endeble, por no decir inexistente. Podríamos reducir estas situaciones, por un lado, a aquellos hogares que acogen a parientes pobres ${ }^{18} \mathrm{o}$ a otras personas, aunque los casos que hemos localizado, en este sentido, suelen corresponderse con familias acomodadas. Por otro, a todos aquellos legados que aparecen en los

${ }^{18}$ D. Juan Rosón, casado y con cinco hijos, tiene en su casa a una tía, de sesenta años, pobre y dos hijos de ésta. A.CH.V. Sec. P. y P. C. 147-2. Domingo Alonso, casado y con dos hijos, mantiene en su casa a una mujer cuyo marido está ausente. Francisco Rodríguez, mantiene a un pobre de solemnidad. Santiago Fernández tiene en su casa a dos cuñadas, una "simple" y la otra "impedida". A.H.P.L. C. 8001 y 8002 
testamentos dirigidos a parientes, en cualquier grado, $\operatorname{criados}^{19}, \operatorname{vecinos}^{20} \ldots$ a los que el presente que les dejan suele ir acompañado de cláusulas tales como "por el mucho amor", "por los cuidados que me prestó"... Lo cual, nos demuestra que los lazos afectivos iban más allá del núcleo familiar ${ }^{21}$. Pero cuando entramos en el terreno donde los cuidados que se van a prestar son a cambio de unos bienes económicos más cuantiosos, surge ese interrogante: ¿comprar asistencia?. En este caso, la documentación notarial es mucho más rica. Las atenciones que requieren son previamente consensuadas entre la partes, para acudir posteriormente al notario y canjearlas por un mejora, o una donación, etc.

Respecto a la asistencia que los padres solicitaron de sus hijos, la respuesta la encontramos en dos escrituras: la dote, normalmente masculina, y los testamentos. Ambas se corresponden con dos variables demográficas transcendentales en la vida: la nupcialidad y la muerte. El matrimonio de uno de los hijos, fundamentalmente el del varón primogénito, constituía un hecho fundamental para la reestructuración del hogar, en caso de que los padres solicitarán de él atención y cuidados para su vejez. O también podía suceder que la iniciativa la tomara el hijo, que se ofrece a continuar bajo la autoridad paterna a cambio de ocupar un lugar preferente en la herencia. Por su parte, la defunción será otro momento decisivo para conocer el tema de los cuidados a los mayores. Pero en este caso, y teniendo en cuenta la proximidad temporal que frecuentemente solía haber entre el testamento y el fallecimiento del testante, los cambios que provocarían en el seno de la familia no serían tan duraderos. Atendiendo a las circunstancias en que se llevan a cabo ambas escrituras podemos diferenciar dos tipos de concepción de la asistencia. Mientras las primeras, sino mediaba de forma inmediata ningún acontecimiento de tipo luctuoso, dan al hogar una estabilidad más prolongada y con clara vocación asistencial. Las segundas, salvo aquellos casos que sirven para corregir una decisión anterior, valen para premiar unos cuidados que se venían practicando desde la afectividad y sin que mediara contrato o para encargar el cuidado del cónyuge que queda vivo, el resto podríamos encuadrarlas más en el

${ }^{19}$ María de Lama, que falleció sin hijos, dejó a una criada, diferentes bienes inmuebles y raíces. A.H.P.L. C. 6575

${ }^{20}$ Isabel Álvarez, deja ropa a una vecina, en 1762, "por las muchas atenciones que le prestó". A.H.P.L. C. 6635 Por el mimo motivo, un año después, María Álvarez, legó a otra vecina, ropa y una res de ganado vacuno. A.H.P.L. C. 6636

${ }^{21}$ Sobre los lazos de vecindad, Vid. CHACÓN JiMÉNEZ, F., (1987): "Notas para el estudio de la familia en la región de Murcia durante el Antiguo Régimen". En VILAR, P.(Coord.). La familia en la España Mediterranea: (Siglos XV-XIX), Barcelona: Crítica, pp. 129- 171. 
terreno económico. Es decir, en el deseo de los padres, dentro de los marcos legales, de conservar y proyectar la unidad de producción que habían logrado crear.

En cuanto a la dote masculina, que suele recogerse en la misma escritura que realizan los padres de su futura esposa, hemos de considerarla, prácticamente en todos los casos que aparece, como una promesa de mejora. En ellas al varón le ofrecen ser preferenciado con el Tercio y el Remanente del Quinto, a cambio de una residencia continuada ${ }^{22}$ en la casa paterna y del trabajo mancomunado. De esa norma se salen, aunque no son muy frecuentes esas cláusulas en las escrituras, aquellas familias que previniendo los conflictos que se pudieran crear entre ambas generaciones $^{23}$, señalan unos bienes para el sostén de la nueva pareja y una casa, normalmente aledaña, en la que podría establecerse ${ }^{24}$. El contenido de estas excepcionales disposiciones nos ratifica, una vez más, que tampoco en el Concejo de Laciana la carta de dote masculina implicaba una transferencia de bienes, al reservarse los dotadores la autoridad sobre ellos. Con ese control que mantiene la pareja anciana sobre los bienes pretende asegurarse el que no se escindiera la corresidencia y el recibir los auxilios que requerían, ya que si los hijos incumplen la responsabilidad adquirida perderían el acceso a tan sustanciosa oferta.

Otra de las fuentes que puede darnos contestación a esos auxilios que solicitan los padres para su ancianidad, son las escrituras testamentarias ${ }^{25}$. Éstas, además de constituir por si solas un elemento de información, ya que no todo el mundo estuvo dispuesto a adelantar este tipo de decisiones, también nos facilitan datos sobre si los hijos que en el momento de contraer matrimonio recibieron la promesa de

${ }^{22}$ Lo que nos demuestra que los progenitores no estaban dispuestos a aceptar la ruptura de la corresidencia. Respecto a las ventajas, para predecesores y sucesores, de que la nueva pareja inicie una vida independiente, vid. NAROTZKY, S.: (1991): "La renta del afecto: ideología y reproducción social en el cuidado de los viejos", En Prat, J., Martínez, U., Contreras, J. y Moreno, I. (eds.) Antropología de los pueblos de España. Madrid: Taurus , pp. 464-474.

23 Cuando contraen matrimonio, en 1762, D. José Flórez Sierra y Doña Ramona Carvallo, él recibe la promesa de ser mejorado en el Tercio y remanente del Quinto, pero en caso de no poder compartir techo con sus padres, éstos se comprometen a entregarles media casa, para que vivan, a cambio de que la nueva pareja les entregue cuatro reales diarios para su manutención. A.H.P.L. C. 6783.

24 Todo ello sin que tenga por qué romperse el compromiso adquirido por ambas partes. FERNÁNDEZ CORTIZO, C., (1988): “En casa y compañía: grupo doméstico y estrategias familiares en la Galicia Occidental... pp. 145-165.

${ }^{25} \mathrm{Al}$ igual que en Cataluña, muerte y matrimonio, son los momentos clave en el proceso de transmisión "de los derechos sobre la propiedad NAROTZKY, S.: (1991): "La renta del afecto: ideología y reproducción social en el cuidado ...., pp. 464-474 
mejora, a cambio de asistencia, respondieron favorablemente a la obligación adquirida. En principio, parece que estamos ante una descendencia disciplinada. Son muy pocos los casos que hemos encontrado en que los padres se retracten ${ }^{26} \mathrm{de}$ la decisión tomada años antes ${ }^{27}$. Pero tampoco hemos de olvidar que, según el hipotético cálculo que hemos realizado para elabora el organigrama, no eran muchos los años de convivencia, lo cual atenuaría mucho las fricciones que pudieran surgir entre ambas generaciones. También hubo casos intermedios, la promesa de mejora no tuvo lugar cuando el hijo contrajo matrimonio, ni tampoco se esperó al testamento. Entonces el mecanismo al que se recurría era la Escritura de Mejora ${ }^{28}$.

En el siguiente cuadro hemos reflejado el tipo de reparto por el que se inclinaron los montañeses, tomando como referencia el sexo y estado civil. Nos hemos ceñido a las personas que en algún momento de su vida matrimoniaron (cuadro $n^{\circ} 3$ ).

El tipo de reparto por el que se inclinaron los padres ya nos da idea de si habían acogido, o no, a alguno de sus descendientes, para que les atendiera durante la senectud y para que en el mismo hogar tuviera lugar la reproducción social. Pero la finalidad de esta práctica, como nos han demostrado algunos testamentos, no se ciñe exclusivamente a ese interés, ya que dentro de los grupos más pudientes ${ }^{29} \mathrm{el}$ deseo de prolongar el rango económico ${ }^{30}$ y social $^{31}$ a través de alguno de sus

${ }^{26}$ Entre ellos estaría el de Pedro García, el cual anula la Mejora que mandó a su hijo cuando se casó "porque se fue a vivir a otro lugar y no se ocupó de ellos ni de sus hermanos". A.H.P.L. C. 6730. Lo mismo hizo María Rodríguez. En este caso, porque el hijo, además de no vivir con ella, tampoco hizo frente al compromiso de pagar unas deudas maternas. A.H.P.L. C. 6717. Por su parte, Juan Martínez, no nos señala explícitamente el motivo que le llevó a anular una mejora hecha unos años antes, pero suponemos no sea muy distinto a los anteriores. A.H.P.L. C. 6672.

${ }^{27}$ En el caso contrario, es decir confirman la promesa de mejora "por cumplir bien el cuidado de sus padres", están, entre otros muchos, Antonio Mallo, Juan Álvarez, Francisca López, etc. A.H.P.L. C. 2549.

28 Por ejemplo, en 1761, Francisco Feito, recibió una escritura de Mejora de sus padres, en compensación por haberse trasladado, junto a su esposa e hijos, al domicilio paterno. A.H.P.L. C. 6634.

29 "tengamos en cuenta que familias, redes sociales, linajes, clientelas, son algo más que formas de organización en tanto que inciden sobre el orden político y los procesos de movilidad social". CHACÓN JimÉnEZ, F., (2001): "Historia de grupos: parentesco, familias, clientelas ... pp. 165-183.

${ }^{30}$ Catalina Álvarez, mejoró a su nieto Miguel, con la condición de que esos bienes habían de pasar siempre juntos a un solo heredero. A.H.P.L. C. 6712. 
vástagos $^{32}$, o la necesidad de mano de obra, fueron causas coadyuvantes a la hora de escoger un modelo hereditario u otro.

Cuadro $n^{\circ} 3$. El reparto de la herencia

\begin{tabular}{|c|c|c|c|c|c|c|c|c|c|}
\hline \multicolumn{10}{|c|}{ A) Montaña noroccidental leonesa" } \\
\hline & & & & $(3$ & & $(4$ & & $(5$ & \\
\hline & (1) & (2) & (3) & $N^{o}$ & $\%$ & $N^{o}$ & $\%$ & $N^{o}$ & $\%$ \\
\hline \multicolumn{10}{|c|}{ Testamentos masculinos } \\
\hline Casado & 99 & 87 & 80 & 36 & 45,0 & 21 & 26,3 & 23 & 28,8 \\
\hline Viudo & 27 & 24 & 22 & 12 & 54,5 & 6 & 27,3 & 4 & 18,2 \\
\hline Total & 126 & 111 & 102 & 48 & 47,1 & 27 & 26,5 & 27 & 26,5 \\
\hline \multicolumn{10}{|c|}{ Testamentos femeninos } \\
\hline Casado & 51 & 30 & 28 & 10 & 35,7 & 16 & 57,1 & 2 & 7,1 \\
\hline Viudo & 81 & 68 & 57 & 22 & 38,6 & 29 & 50,9 & 6 & 10,5 \\
\hline Total & 132 & 98 & 85 & 32 & 37,6 & 45 & 52,9 & 8 & 9,4 \\
\hline \multicolumn{10}{|c|}{ Total testamentos } \\
\hline Casado/a & 150 & 117 & 108 & 46 & 42,6 & 37 & 34,3 & 25 & 23,1 \\
\hline Viudo/a & 108 & 92 & 79 & 34 & 43,0 & 35 & 44,3 & 10 & 12,7 \\
\hline Total & 258 & 209 & 187 & 80 & 42,8 & 72 & 38,5 & 35 & 18,7 \\
\hline \multicolumn{10}{|c|}{ B) Concejo de Laciana } \\
\hline $1710-1830$ & 188 & & 167 & 61 & 36,5 & 51 & 30,5 & 55 & 32,9 \\
\hline
\end{tabular}

(1), $N^{o}$ Testamentos; (2), Con hijos; (3), Más de un heredero; (4), Mejora larga; (5), Mejora corta;

(6), Igualitario

En la Montaña Noroccidental leonesa, hubo una preferencia hacia la mejora, ya fuera "mejora larga",33, que aglutinaba el Tercio y remanente del Quinto -42,8\%-, o la "mejora corta" -38,5\%-, que se reducía a algún legado. Aunque es muy frecuente

31 Antonio Díez de la Torre, dejó como heredero a un hijo, en previsión de que éste pudiera fallecer, la herencia pasaría a un nieto, y si éste también falleciese, entonces a una nieta, pero con la condición de que había de casarse con un primo, o con una persona de su estado, sino perdería todo derecho sobre esos bienes. A.H.P.L. C. 6782. Antonio González, por su parte, manda a sus hijas Pacuala y Francisca, que, además de las legítimas que les correspondan, escojan una vaca cada una. Pero en caso de que contrajeran matrimonio con una persona que no perteneciera a la hidalguía deberían volver ese ganado al cuerpo de bienes. A.H.P.L. C. 6785. Pedro García y Josefa Luengo, mejoran a un hijo, poniendo como condición que si esa herencia llegara a recaer en una persona del Estado General, entonces pase a otro descendiente. A.H.P.L. C. 6782.

32 Esta forma de aprovechar la mano de obra familiar encuadraría perfectamente en la afirmación hecha por WOLF, E.R. (1999). "Relaciones de paentesco, de amistad y patronzgo en las sociedades complejas",. En VVAA. Antropología Social de las Sociedades Complejas. Barcelona: Alianza, pp. 19-39, p. 25. "Además de funcionar con la máxima eficacia y el mínimo costo, la familia se adapta óptimamente a los cambios de las condiciones que defienden y delimitan su existencia".

33 SAAVEdRA FERnÁNDEZ, P., (1988): “Casa y comunidad en la Galicia interior... pp. 85-143. 
encontrar testamentos ${ }^{34}$ donde aparecían la "mejora larga" a algún hijo y un pequeño presente a otro de los descendientes ${ }^{35}$, de ahí que éstas llegaran a estar presentes en el $74,6 \%$ de los testamentos montañeses ${ }^{36}$. El reparto igualitario, por su parte, fue la opción de tan sólo del $18,7 \%{ }^{37}$ de los testadores (cuadro ${ }^{\circ} 3$ ).

Ahora bien, si realizamos un estudio diferencial por sexos y estado civil observamos que el comportamiento es muy diferente. El más inclinado hacia comportamientos patrilocales fue el hombre, guiado muchas veces por motivaciones económicas. El varón prolongaba el apellido y la casa, en el sentido inmaterial $^{38}$, y además traía una mujer, que era en realidad la que tomaba las riendas asistenciales ${ }^{39}$. Dentro de ese colectivo masculino, fueron los viudos, sin una pareja en la que apoyar sus expectativas ante la ancianidad, o posiblemente como compensación a la asistencia que ya estaban disfrutando, los que más se volcaron en mejorar a uno de sus descendientes, a fin de asegurarse continuar recibiendo el auxilio necesario en los momentos finales de su vida. Los casados aparecen a cierta distancia, nueve puntos menos. Quizás el mantener la presencia femenina a su lado, o que a través de la figura de ésta se prolongara el hogar, fue lo que los condujo a retraerse un poco en el trato preferencial a uno de sus hijos. Además también encontramos codicilos en los que estos hombres dejan que sean sus esposas quienes, en función del comportamiento de sus hijos, escojan al que consideraran más apto para prolongar el tronco familiar en el seno de la misma casa.

34 Para la elaboración del cuadro nosotros hemos optado por resumir todas esas situación a tres: los que optaron por la "mejora larga", independientemente de que en esos testamentos aparezca algún legado; la "mejora corta"; y el reparto igualitario.

35 Sirva como ejemplo, para clarificar ésto, el testamento de Doña Josefa Carballo. Esta mujer tenía 4 hijos, dos varones y dos mujeres. En su testamento mejora a uno de los varones con el Tercio y Remanente del Quinto, y, aparte, mandó cinco reses vacunas a una de sus hijas y un ternero a una nieta. A.H.P.L. C. 6604.

${ }^{36}$ PÉReZ Alvarez, M.J. (1996). La Montaña Noroccidental leonesa.... p. 356

37 Esta cifra, aunque ligeramente más baja, es muy similar a la que nos ofrece FERNÁNDEZ Cortizo, C., (1988): "En casa y compañía: grupo doméstico y estrategias familiares en la Galicia Occidental...pp. 145-165., para la Galicia occidental. La diferencia entre ambos territorios radica en que el desigualitarismo allí estuvo mediatizado por las "mejoras cortas", mientras en esta zona leonesa fue la "mejora larga" la que más adeptos tuvo.

${ }^{38}$ Comas D'Argemir, M.D., (1991): "Casa y comunidad en el Alto Aragón. Ideales culturales y de reproducción social”, Revista de Antropología Social, $n^{\circ}$ 0, pp. 131-150.

${ }^{39}$ NAROTZKY, S.: (1991): "La renta del afecto: ideología y reproducción social en el cuidado...., pp. 464-474. 
En cuanto a las mujeres, también la elección sucesoria dominante fue la mejora, pero la "mejora corta". Dentro de este grupo, las más aficionadas a recurrir al Tercio y Remanente del Quinto fueron las viudas. Esa decisión estaría motivada, al igual que en el caso de los viudos, por el temor a la soledad o para premiar la asistencia recibida durante la viudedad. Sin olvidar que algunos cónyuges derivaron en ellas la responsabilidad de realizar ese tipo de reparto. Por otro lado, hemos de tener presente que a esa circunstancia, la mujer se adapta mejor que el varón, de ahí que no se alcancen porcentajes tan elevados como en el sexo contrario $^{40}$. Entre las casadas es donde se aprecia las cifras más bajas de ese tipo de repartos. Posiblemente dejaran que la decisión final recayera en el cónyuge que quedaba. Lo cual cooperará a esos elevados porcentajes de "mejora larga" que se constata en los hombres viudos. Pero si hubo algo por lo que destacó el testamento femenino fue, como hemos señalado, por la "mejora corta", la cual aparece en el $52,9 \%$ de los testamentos en los que hay hijos. De todas formas, cuando se trataba de bienes raíces éstos no fueron desperdigados fuera del tronco familiar. Ese porcentaje de legados, en cualquiera de los cuatro casos puede ser más elevado, debido, como ya hemos dicho, a que en numerosas ocasiones se combinaron ambas mejoras.

Pero ¿qué ocurrió con todos aquellos matrimonios que optaron por el reparto igualitario, o formas de residencia neolocal para sus hijos? ¿fueron personas desatendidas y olvidadas por sus sucesores?. Más aún cuando la precariedad también les venía por el plano económico, ya que los mayores porcentajes en este tipo de opción hereditaria coinciden con las explotaciones de menor tamaño ${ }^{41}$. Creemos que en este punto es donde, al no tener cabida los mecanismos de tipo económico, no quedaría otra alternativa que los lazos de solidaridad. Las redes asistenciales se tupirían en base a una distribución de funciones y responsabilidades entre los hijos para prestar socorro a los progenitores. Lo cual no sería muy complicado si tenemos en cuenta que estamos ante pequeñas comunidades rurales en las que no existe la distancia ${ }^{42}$. No queremos, con este planteamiento, decir que los más pudientes que optaron por el reparto igualitario vieran como sus hijos los relegaban, pues no dudamos que el comportamiento de estos descendentes fuera similar al de sus vecinos más pobres.

\footnotetext{
${ }^{40}$ Gomila GraU, M.A. (1995): "Mecanismos de organización social a través de la familia...., p. 654.

41 Pérez Álvarez, M. J., (2004): "Familia y estrategias familiares en el marco de unas estructuras socioeconómicas tradicionales... pp. 121-147

42 Gomila Grau, M.A. (1995): "Mecanismos de organización social a través de la familia .... p. 661.
} 
Pero no sólo los que tenían hijos solicitaban asistencia para los últimos años de su vida. Hay otros colectivos que podían llegar a encontrarse desamparados: huérfanos, enfermos.... En esos casos, dependiendo de la circunstancia individual de la persona necesitada, la "lógica reproductiva" ${ }^{43}$ desarrolló unos mecanismos u otros, pero, en cualquier caso, o al menos así lo demuestra la documentación, éstos siempre tuvieron una fuerte carga económica. Sin olvidar que el ámbito de la solidaridad, como mecanismo asistencial, no se escrituraba, e incontestablemente existiría en esas sociedades al igual que en las relaciones paterno-filiales. Varias son las situaciones que podríamos diferenciar.

Había personas jóvenes con enfermedades que les hacían dependientes. En esta situación, eran los padres, quienes, ante el temor de que esos hijos fueran abandonados, buscaban una solución. Solución ésta que no solían demorar, debido, como hemos visto en el organigrama, a que teóricamente el tiempo que pasaba entre el matrimonio del primer hijo y el fallecimiento del progenitor era de unos cuatro años. Margen temporal muy pequeño, si además se tiene en cuenta que la sombra de la muerte siempre estaba al acecho. La forma de protección que consideraron más adecuada, para que los descendientes desvalidos recibieran esa asistencia, fue dejarlos bajo la custodia de un hermano o pariente próximo ${ }^{44}$, a cambio de una promesa de mejora. De ahí, que ésta se escriturara cuando el hermano/a del incapacitado, escogido para asumir la responsabilidad, se casaba ${ }^{45}$. Aunque también hubo casos en los que se esperó hasta el momento de testar.

El mismo sentimiento, lazos de afectividad para con los hijos que se consideraban más desamparados, hijos pequeños ${ }^{46}$, hijas solteras ${ }^{47} \ldots$, es lo que

${ }^{43}$ Comas D'Argemir, M.D., (1991): “Casa y comunidad en el Alto Aragón. ... pp. 131-150.

44 Una mujer viuda, de Toreno, mejoró a una sobrina, cuando se casó, para que en contraprestación se hiciera cargo de su hijo, "falto de entendimiento". A.H.P.L. C. 2357.

45 José Álvarez, recibió en dote la promesa de la Mejora del Tercio y remanente del Quinto, pero a cambio debía hacerse cargo de sus dos hermanos solteros y mudos. A.H.P.L. C.6720. Por su parte, y en la misma situación encontramos a Manuel de la Parte, el cual tendría su cargo una hermana incapacitada. A.H.P.L. C. 6633.

${ }^{46}$ Pedro de Villablino, padre de tres hijos, legó a la hija pequeña, algunos bienes raíces; lo mismo hizo Domingo García, con su hija pequeña; o un hombre, de Toreno, "por ser una niña y estar por criar". A.H.P.L. C. 6649.

47 Alonso Martínez, padre de cinco hijos, en 1707, dejó a uno de los varones la casa y el hórreo, pero no el pajar, que quedaba en reserva por si lo necesitaba alguna hija que pudiera verse desamparada. Domingo Calzón y María Peredillo, padres de dos hijos, detraen de la mejora que prometen al primero, parte de la casa, por si la necesitara la hija que está soltera. A.H.P.L. C. 6571 y 2349. 
movería a muchos padres a dedicarles una consideración especial en su testamento. Más extremo era el caso de los niños que quedaban huérfanos de padre y madre, entonces se recurría a los tíos ${ }^{48}$ o parientes próximos para que los acogieran ${ }^{49}$

Respecto a los solitarios/as, buscaban en un pariente la cobertura necesaria a cambio de la herencia. El contrato lo materializaban en una escritura de donación ${ }^{50}$, de dote, o bien, en un testamento que se hacía de forma anticipada, es decir, no se esperaba a los momentos finales de la vida para plasmar la voluntad. Esa premura en registrar las voluntades se debería al interés, por ambas partes: unos, asegurarse que la muerte repentina de los donantes no les apartara del disfrute de los bienes que les tenían apalabrados; y los otros, que esa incertidumbre no repercutiera en el digno cuidado y enterramiento que esperaban recibir. En cualquier caso, los solitarios, al igual que los que tenían descendencia, exigían compartir morada, efectuándose el traslado de la pareja joven, o del pariente sin más, a su casa, o a la inversa. También los miembros de este colectivo eran previsores en lo referente a desprenderse de la propiedad jurídica de los bienes ${ }^{51}$, la cual reservaban hasta el momento final de su vida. Estos nuevos grupos los encontramos en la categoría de familia extensa, si lo que aprovecharon para presentarse ante el notario fue un futuro casamiento. Paralelamente a esas situaciones, surgieron aquellas otras en las que la decisión no se tomaba ante un inminente matrimonio, sino que simplemente se hacía una escritura de donación, con la misma finalidad que en le caso anterior. Entonces ese nuevo grupo doméstico lo encontramos entre las familias desestructuradas, donde es fácil localizar célibes en compañía de sobrinas/os, primos... Esas donaciones, que solían recaer en mujeres ${ }^{52}$ que no habían sido mejoradas por sus padres, supusieron, en el campo económico, regenerar las

48 Juan Álvarez del Campillo, viudo con dos hijos y una nuera, en 1718, acogía en su casa a una sobrina pobre, enferma y huérfana, a la cual dice mantener. A.G.S. Sec. P. y P. C. 147-2. Antonio Sabugo, en 1752, tiene bajo su amparo a dos sobrinas huérfanas. A.H.P.L. C. 8579.

49 Sobre la incorporación de estos niños al hogar de unos parientes, vid. Gomila GraU, M.A. (1995): "Mecanismos de organización social a través de la familia ... pp. 653-673.

${ }^{50}$ María García, de Montrondo, impedida de pies y manos, donaba, en 1750, sus legítimas a sus hermanos, a cambio de su protección. A.H.P.L. C. 6784.

${ }^{51}$ María Álvarez, de Villanueva, "soltera mayor e incapacitada", donó todos sus bienes a Pedro Iglesias, a cambio de que la cuide y alimente, pero se reserva el poder de vender lo que necesitase a lo largo de su vida. A.H.P.L. C. 6784. Manuel Llama hacia una escritura de donación a favor de su cuñado, Matías Hidalgo, de todos sus bienes a cambio de que lo asista, y si no pueden vivir juntos, Matías dará a Manuel, cada año, 100 reales, 15 cantaras de vino, 6 cargas de pan y 6 varas de paño pardo. A.H.P.L. C. 6785.

52 PÉrez Álvarez, M. J., (2004): "Familia y estrategias familiares en el marco de unas estructuras socioeconómicas tradicionales... pp. 121-147 
condiciones de reproducción social de esas jóvenes, aunque excepcionalmente las situaría en posiciones ventajosas, ya que los colectivos de solitarios/as no eran los que más bienes acumulaban. Así mismo, tales donaciones en algunos casos significaron la prolongación integra ${ }^{53}$ o parcial $^{54}$ de los patrimonios paternos, ya que era frecuente que a esos herederos se le pusiera la condición de renunciar a la herencia que pudieran corresponderles de sus progenitores. Pero no siempre estas personas sin descendencia buscaban prestaciones para sí, en muchas ocasiones el objeto de preocupación eran sus hermanos. Pero a diferencia de los casos anteriores, la mayor parte de las escrituras de este tipo que hemos encontrado correspondían a personas jóvenes y huérfanas ${ }^{55}$.

De todo lo expuesto hasta aquí, se deduce que, al margen de la solidaridad familiar que pudo existir para con los más débiles, uno de los mecanismos a los que más se recurrió para proteger a esos grupos, fue el de la compensación económica para con las personas destinadas a ejercer esa responsabilidad. Esa se ponía en marcha en una escritura de dote, donación, mejora o testamento. Los receptores de la misma, solían ser descendientes directos, hijos o, en ausencia de éstos, sobrinos. A partir de ese momento se producía un reajuste familiar, con formación de nuevos núcleos domésticos o desplazamientos de domicilio, cuya finalidad era satisfacer las expectativas de los implicados en las escrituras.

${ }^{53}$ Polonia Rodríguez, tía de Rafael y José Rodríguez, dejó como heredero, de un considerable patrimonio, a José, a cambio de que éste renunciara a la sustanciosa parte que le pudiera corresponder de su herencia paterna y materna, la cual pasaba íntegra a Rafael. A.H.P.L. C. 6598.

${ }^{54}$ Es el caso, por ejemplo, de un vecino de Sosas de Laciana, que en 1764, dejó como heredera a una sobrina a condición de que cuide de él y de su esposa y "no pida nada de las legítimas que de sus padres que puedan corresponder". No sabemos el número de hermanos de esta mujer, pero, en cualquier caso, el ser uno menos a repartir suponía un menor fraccionamiento del patrimonio acumulado por sus padres. A.H.P.L. C. 6635.

${ }^{55}$ Francisca Álvarez, soltera, deja como heredero a su hermano, y lega 100 ducados a su hermana si se casa, y "200 ducados si no llegara a tomar estado". Bernardo Álvarez Carvallo, soltero, nombra como heredero a uno de sus hermanos, pero le pone como condición que ha de cuidar del resto de hermanos "hasta que se acomoden". A.H.P.L. C. 6730 